\title{
Diffusion quantum Monte Carlo calculation of the quasiparticle effective mass of the two-dimensional homogeneous electron gas
}

\author{
N. D. Drummond \\ Department of Physics, Lancaster University, Lancaster LA1 4YB, United Kingdom \\ R. J. Needs \\ TCM Group, Cavendish Laboratory, University of Cambridge, J. J. Thomson Avenue, Cambridge CB3 OHE, United Kingdom
}

(Received 30 August 2012; revised manuscript received 21 December 2012; published 31 January 2013)

\begin{abstract}
The quasiparticle effective mass is a key quantity in the physics of electron gases, describing the renormalization of the electron mass due to electron-electron interactions. Two-dimensional electron gases are of fundamental importance in semiconductor physics, and there have been numerous experimental and theoretical attempts to determine the quasiparticle effective mass in these systems. In this work we report quantum Monte Carlo results for the quasiparticle effective mass of a two-dimensional homogeneous electron gas. Our calculations differ from previous quantum Monte Carlo work in that much smaller statistical error bars have been achieved, allowing for an improved treatment of finite-size effects. In some cases we have also been able to use larger system sizes than previous calculations.
\end{abstract}

DOI: 10.1103/PhysRevB.87.045131

PACS number(s): 73.20.-r, 71.10.Ay, 02.70.Ss

\section{INTRODUCTION}

Two-dimensional (2D) electron gases are ubiquitous in modern semiconductor devices. Surprisingly, however, there remain significant gaps in our knowledge of the properties exhibited by these fascinating systems. In recent years experimentalists have realized increasingly high-quality (low-disorder) 2D homogeneous electron gases (HEGs) at low densities in quantum-well structures ${ }^{1,2}$ and field-effect transistors. ${ }^{3}$ As the density is lowered, correlation effects play an ever more important role, and it is hoped that the resulting exotic behavior could be exploited in a new generation of electronic and spintronic devices. At very low densities the Coulomb repulsion between the electrons dominates, and the HEG forms a Wigner crystal. ${ }^{4-7}$ At higher densities the kinetic energy dominates and the electrons form a Fermi fluid, in which most properties are qualitatively (and at high densities, quantitatively) similar to those of a free-electron gas. Fermi liquid theory ${ }^{8,9}$ is the phenomenological framework within which the normal behavior of Fermi fluids is understood.

According to Fermi liquid theory, low-lying excitation energies in HEGs are free-electron-like, and the effects of interactions are encapsulated in (i) a renormalization of the electron mass (the quasiparticle effective mass) and (ii) a set of parameters describing the interaction of pairs of excited quasiparticles. In this work we use quantum Monte Carlo (QMC) methods to determine the quasiparticle effective mass by calculating single-particle excitation energies as differences in the total energy when electrons are either added to or removed from the ground state.

In our calculations we have used the variational Monte Carlo (VMC) and diffusion Monte Carlo (DMC) methods. ${ }^{10}$ In the VMC method we take the expectation value of the many-electron Hamiltonian with respect to a Slater-Jastrowbackflow trial wave function, ${ }^{11-13}$ which is optimized by minimizing first the variance of the energy, ${ }^{14,15}$ and then the energy expectation value ${ }^{16}$ with respect to free parameters in the wave function. In DMC calculations ${ }^{17}$ we simulate a population of "walkers" whose dynamics are governed by the
Schrödinger equation in imaginary time in order to project out the ground-state component of an initial wave function. The fixed-node approximation ${ }^{18}$ is used to impose fermionic antisymmetry. All our QMC calculations were performed using the CASINO code. ${ }^{19}$

In Ref. 20 we presented a DMC calculation of the $2 \mathrm{D}$ HEG single-particle energy band, enabling us to predict the quasiparticle effective mass. In the present work we have had access to the Jaguar machine at Oak Ridge Leadership Computing Facility, enabling us to achieve higher accuracy in our DMC calculations, and leading to a refinement of our earlier work.

The rest of this paper is structured as follows. In Sec. II we give an overview of the relevant aspects of Fermi liquid theory. In Sec. III we describe our computational approach. Our results are presented in Sec. IV. Finally, we draw our conclusions in Sec. V. We use hartree atomic units, in which the Dirac constant, the electronic charge and mass, and $4 \pi$ times the permittivity of free space are unity $\left(\hbar=|e|=m_{e}=\right.$ $\left.4 \pi \epsilon_{0}=1\right)$, throughout.

\section{LANDAU ENERGY FUNCTIONAL}

\section{A. Parametrization of excitation energies}

According to Fermi liquid theory the total energy of a HEG in a particular excited state is given by the Landau energy functional $^{9}$

$$
\begin{aligned}
E= & E_{0}+\sum_{\mathbf{k}, \sigma} \mathcal{E}_{\sigma}(\mathbf{k}) \delta \mathcal{N}_{\mathbf{k}, \sigma} \\
& +\frac{1}{2} \sum_{(\mathbf{k}, \sigma) \neq\left(\mathbf{k}^{\prime}, \sigma^{\prime}\right)} f_{\sigma, \sigma^{\prime}}\left(\mathbf{k}, \mathbf{k}^{\prime}\right) \delta \mathcal{N}_{\mathbf{k}, \sigma} \delta \mathcal{N}_{\mathbf{k}^{\prime}, \sigma^{\prime}},
\end{aligned}
$$

where $\delta \mathcal{N}_{\mathbf{k}, \sigma}$ is the change to the ground-state quasiparticle occupation number for wave vector $\mathbf{k}$ and spin $\sigma$, and $E_{0}$ is the ground-state energy. The energy band $\mathcal{E}_{\sigma}(\mathbf{k})$ is the energy of an isolated quasiparticle. Near the Fermi surface, the energy 
band may be assumed to be linear and hence we may write

$$
\mathcal{E}_{\sigma}(\mathbf{k})=\mathcal{E}_{F}+\frac{k_{F}}{m^{*}}\left(k-k_{F}\right),
$$

where $\mathcal{E}_{F}$ is the Fermi energy, $k_{F}$ is the Fermi wave vector, and $m^{*}$ is the quasiparticle effective mass. The Landau interaction function $f_{\sigma, \sigma^{\prime}}\left(\mathbf{k}, \mathbf{k}^{\prime}\right)$ describes energy contributions arising from pairs of quasiparticles, and will not be considered further in this paper.

The goal of this work is to obtain accurate values for the 2D HEG quasiparticle effective mass $m^{*}$ in the thermodynamic limit at different densities and for different spin polarizations, giving us the most important contribution to the Landau energy functional.

\section{B. Spin-polarization effects}

Both theoretical work ${ }^{20,21}$ and experimental studies ${ }^{1,2}$ have shown that the quasiparticle effective mass has a significant dependence on the spin polarization of the HEG. We have calculated the effective mass for both paramagnetic and ferromagnetic (fully spin-polarized) HEGs. Fully spin-polarized HEGs are experimentally relevant because they may be created by applying an in-plane magnetic field to a 2D electron system. Differences in the quasiparticle effective masses of ferromagnetic and paramagnetic HEGs result in differences in the transport properties, which could be exploited in electronic or spintronic applications, e.g., in devices that use the spin-Coulomb-drag effect. ${ }^{22}$

\section{Finite-size errors}

The 2D HEGs encountered in real devices are sufficiently large that they can be regarded as being of essentially infinite extent. In QMC simulations we can study only small numbers of electrons, however. For a HEG in a finite simulation cell subject to periodic boundary conditions, momentum quantization limits the available wave vectors $\{\mathbf{k}\}$ to a discrete lattice. Furthermore, long-range Coulomb and correlation effects cannot be treated exactly in a finite cell, ${ }^{23,24}$ giving rise to finite-size errors in the energy band and hence effective mass.

Fermi liquid theory is valid only for excitations near the Fermi surface: in this region the quasiparticle lifetime becomes large and hence the quasiparticle momentum occupancies are good quantum numbers. ${ }^{9}$ The energy band is defined by the Landau energy functional at all $\mathbf{k}$, but does not correspond to the quasiparticle band except in the vicinity of the Fermi surface. In the infinite-system limit, the exact energy band is smooth in general and, if the quasiparticle effective mass is well defined, the band must be at least differentiable at the Fermi surface.

\section{QMC CALCULATIONS}

\section{A. Choice of simulation cell}

In all our calculations the simulation cell was square and the simulation-cell Bloch vector ${ }^{25,26}$ was $_{\mathbf{k}_{s}}=\mathbf{0}$. The number of electrons in the ground state was chosen to give a closedshell configuration in each case. For ferromagnetic HEGs, our calculations were performed with $N=29,57$, and 101 electrons in the ground state. For paramagnetic HEGs our calculations were performed with $N=26,50,74,114$, and 162 electrons in the ground state.

The simulation cell was identical for all excitations of a given HEG; hence the electron density increased when electrons were added and decreased when electrons were removed from the ground-state configuration. This procedure results in zero finite-size error for a free-electron gas.

\section{B. Trial wave functions}

We use real, single-determinant trial wave functions for the closed-shell ground states, which is a computationally efficient approach that facilitates the optimization of the wave function. In our QMC calculations we used Slater-Jastrow-backflow trial wave functions. The Jastrow factors consisted of polynomial and plane-wave expansions in the interelectron distances, ${ }^{11}$ while the backflow functions consisted of polynomial expansions in the interelectron distances. ${ }^{13}$ The polynomial expansions were cut off smoothly at the radius of the largest circle that could be inscribed in the simulation cell. The Jastrow factor and backflow function contained a total of 35 and 17 free parameters, respectively, for paramagnetic HEGs, and 27 and 8 free parameters, respectively, for ferromagnetic HEGs. Extrapolation of the VMC energy with different trial wave functions to zero energy variance ${ }^{20}$ suggests that our DMC calculations retrieved more than $99 \%$ of the correlation energy.

For each density, system size, and spin polarization the wave function was optimized in the ground state and the resulting Jastrow factor and backflow function were used in all the excited states, with the exception of a couple of test cases, as discussed in Sec. IV A2.

\section{DMC time steps, populations and equilibration periods}

The DMC time steps used in our calculations were 0.04, 0.2, and 0.4 a.u. at $r_{s}=1,5$, and 10 , respectively, for paramagnetic HEGs, and 0.01, 0.2, and 0.4 a.u. at $r_{s}=1,5$, and 10, respectively, for ferromagnetic HEGs. It was verified that halving the time step had a negligible effect on the energy band: leading-order time-step errors cancel out of the total-energy differences involved. The target population exceeded 1200 configurations in each case, ensuring that population-control bias is negligible.

The number of equilibration steps discarded from the start of each DMC calculation was sufficiently large that the rootmean-square distance diffused by each electron in the equilibration period exceeded the linear size of the simulation cell.

\section{RESULTS}

\section{A. Energy band}

\section{DMC results for the energy band}

The DMC energy band of the paramagnetic 2D HEG, obtained with a Slater-Jastrow-backflow trial wave function, is shown in Fig. 1. Analogous results for a fully ferromagnetic HEG are shown in Fig. 2.

The energy bands calculated in this work at any given system size are in agreement with those calculated in Ref. 20, 


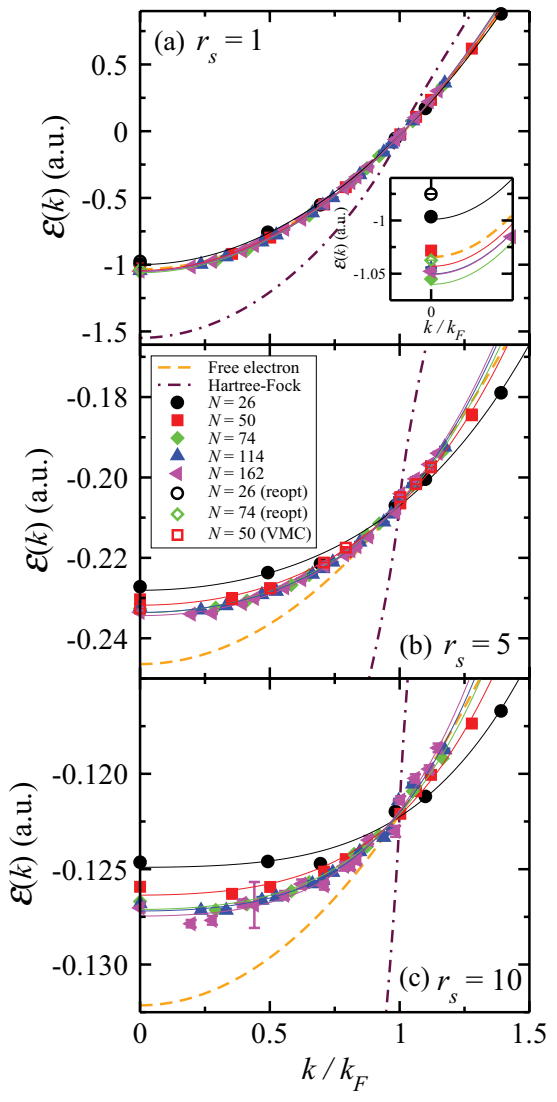

FIG. 1. (Color online) Energy bands $\mathcal{E}(\mathbf{k})$ for paramagnetic 2D HEGs of density parameter (a) $r_{s}=1$, (b) $r_{s}=5$, and (c) $r_{s}=10$ at different system sizes $N$. For the curves labeled "reopt," the wave function was optimized separately in the ground state and excited states. The free-electron and Hartree-Fock bands have been offset so that they coincide with the DMC bands at $k_{F}$. The inset to (a) shows the energy band around $\mathbf{k}=\mathbf{0}$ in greater detail.

but the statistical error bars in the present work are very much smaller due to the considerably larger computational resource available. Furthermore, the random noise in the trial wave function due to optimization by energy minimization with a finite sampling of configuration space is greatly reduced because of the enormous numbers of configurations that were used in the optimizations. As a result it is now possible to discern a systematic trend in the energy band with system size, with the bandwidth tending to increase with system size $N$. This in turn leads to a reduction in the predicted quasiparticle effective mass in the thermodynamic limit, as discussed in Sec. IV B.

\section{Effect of reoptimizing the wave function in excited states}

The excitation of a single electron or pair of electrons has no effect on the optimal Jastrow factor or backflow function in the thermodynamic limit; hence the fact that the Jastrow factor and backflow function can be reoptimized in an excited state in a finite cell is simply a manifestation of finite-size error. Reoptimizing the wave function when an electron was subtracted from $\mathbf{k}=\mathbf{0}$ in a 26-electron HEG at $r_{s}=1$ lowered the DMC energy by 0.000854(4) a.u., reducing the DMC bandwidth and hence increasing the finite-size error, as can be seen for $N=26$ in the inset of Fig. 1(a). In our calculations

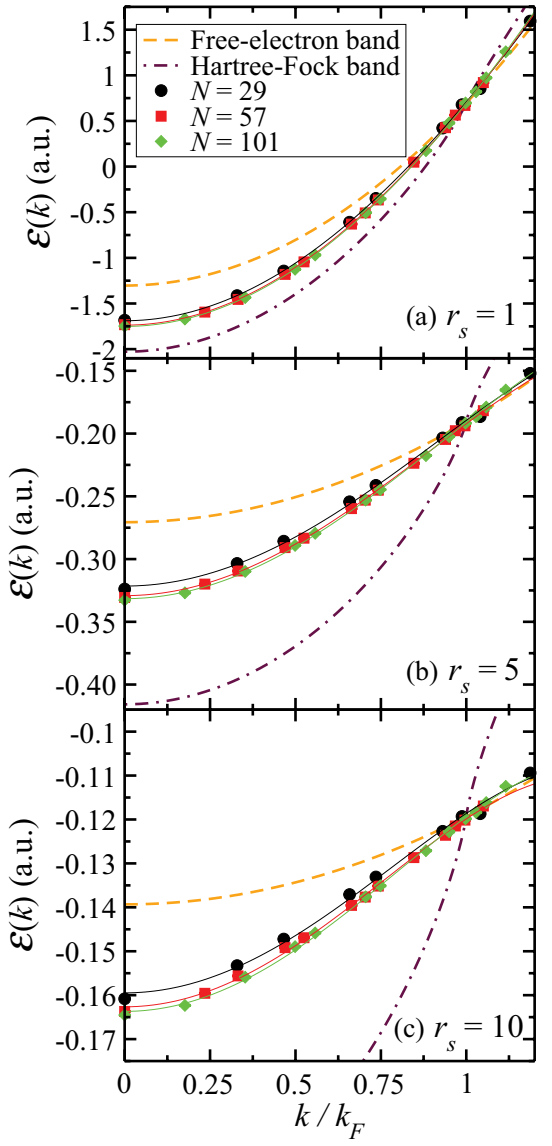

FIG. 2. (Color online) As Fig. 1, but for ferromagnetic HEGs.

we therefore optimized the trial wave function in the ground state and then continued to use the same Jastrow factor and backflow function in our excited-state calculations.

\section{B. Quasiparticle effective mass}

\section{Quartic fits to the energy bands}

At each density, spin polarization, and system size a quartic function $\mathcal{E}(k)=\alpha_{0}+\alpha_{2} k^{2}+\alpha_{4} k^{4}$ was fitted to the DMC energy-band values. The effective mass [defined in Eq. (2)] was then calculated as

$$
m^{*}=\frac{k_{F}}{(d \mathcal{E} / d k)_{k_{F}}} .
$$

We have investigated the dependence of the estimate of the effective mass on the range of energy-band data used to perform the fit. Figures 3 and 4 show the effective mass as a function of the range $\Delta k$ about the Fermi wave vector over which we perform the fit. The figures also show the effective mass when energy-band data from within $\pm 10 \%$ of $k_{F}$ are excluded from the fit. It is clear that the effective mass becomes pathological when $\Delta k$ becomes small (i.e., only excitations in the vicinity of $k_{F}$ are considered), for the reasons discussed briefly in Sec. IV B3 and at length in Ref. 20. As the width of the region over which the fit is performed becomes larger, the effective-mass estimates settle down to well-defined values that behave in a systematic fashion with system size. There is no evidence of any need to exclude data from around 


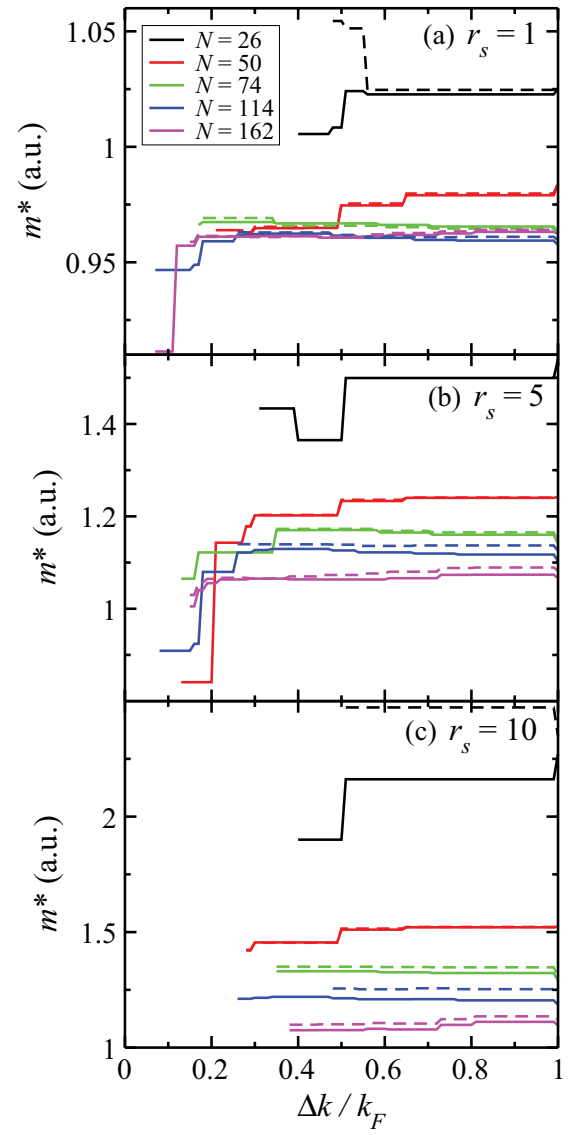

FIG. 3. (Color online) Quasiparticle effective mass $m^{*}$ against range of wave vectors included in the quartic fit of the energy band. Specifically, only wave vectors in the interval $\left[k_{F}-\Delta k, k_{F}+\Delta k\right]$ are used in the fit. For the dashed lines, wave vectors within $10 \%$ of $k_{F}$ are excluded from the fit.

$k_{F}$ however: effectively, the fitting process averages out the pathological behavior in the vicinity of the Fermi surface.

We have compared the VMC and DMC effective masses of a paramagnetic HEG at $r_{s}=5$ and system size $N=$ 50 electrons. The same Slater-Jastrow-backflow trial wave function was used in the VMC and DMC calculations. Despite the fact that the VMC and DMC energy bands differ somewhat, as can be seen in Fig. 1, the VMC and DMC effective masses [1.26(2) and 1.24(3) a.u., respectively] are in excellent agreement. The concurrence between the effective masses obtained with the two different levels of theory indicates that our DMC results at a given system size are reliable.

\section{Derivatives of the energy bands}

Numerical derivatives of the DMC energy-band data, together with the derivatives of the fitted quartic functions and the free-electron and Hartree-Fock bands, are shown in Figs. 5 and 6, for paramagnetic and ferromagnetic HEGs, respectively. The numerical derivatives are evaluated using the central-difference approximation. It can be seen that the derivatives of the bands are generally well behaved, but that the points in the immediate vicinity of the Fermi surface are "outliers." Within Fermi liquid theory, the energy band must have a well-defined derivative at the Fermi surface, indicating

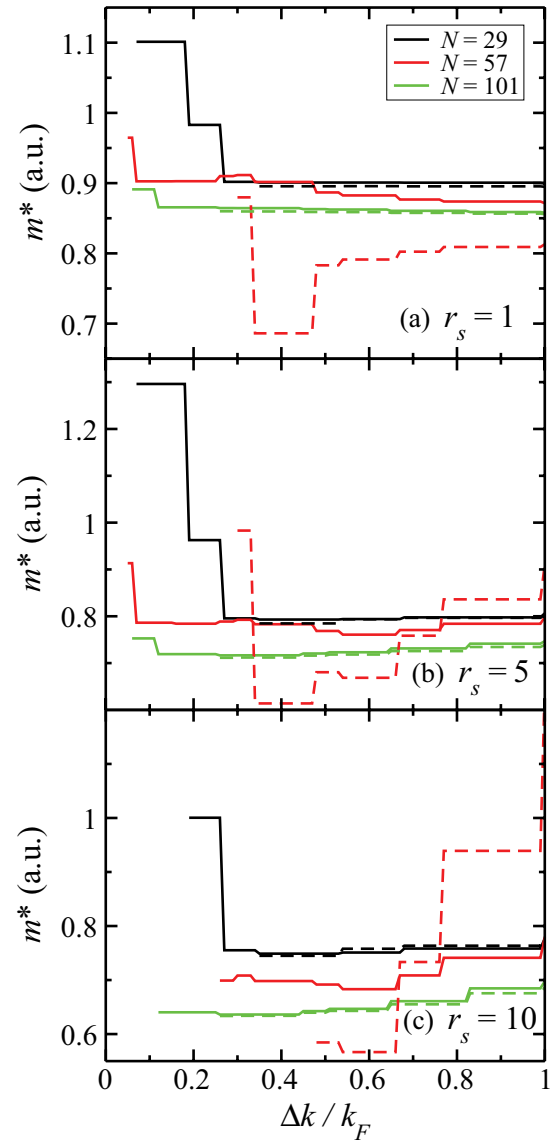

FIG. 4. (Color online) As Fig. 3, but for ferromagnetic HEGs. For $N=57$ electrons, excluding wave vectors within $10 \%$ of $k_{F}$ eliminates all the wave vectors above $k_{F}$ at which energy-band data are available.

that these outliers are unphysical. As argued in Ref. 20, we believe that the pathological behavior in the DMC band at the Fermi surface is associated with the divergence of the derivative of the Hartree-Fock band, because the DMC method retrieves only a finite fraction of the correlation energy. Away from the Fermi surface, the derivatives of the band appear to be smooth (as far as can be seen, given the noise in the data).

\section{Extrapolation to the thermodynamic limit}

The quasiparticle effective masses are plotted against system size in Fig. 7. A systematic trend in the effective mass as a function of system size can be seen. Hence we are able to extrapolate the effective mass to the thermodynamic limit, significantly reducing finite-size errors. The effective-mass values that we report in this work are expected to be more accurate than those reported in Ref. 20.

The finite-size error does not show the $N^{-1 / 4}$ behavior predicted by Holzmann et al. ${ }^{23}$ for excitations near the Fermi surface, presumably because we have fitted to the entire band. (Any $N^{-1 / 4}$ behavior in the band near the Fermi surface is masked by the pathological behavior that is seen in HartreeFock theory and hence QMC calculations. ${ }^{20}$ ) To investigate the behavior of the effective mass as a function of system size, 


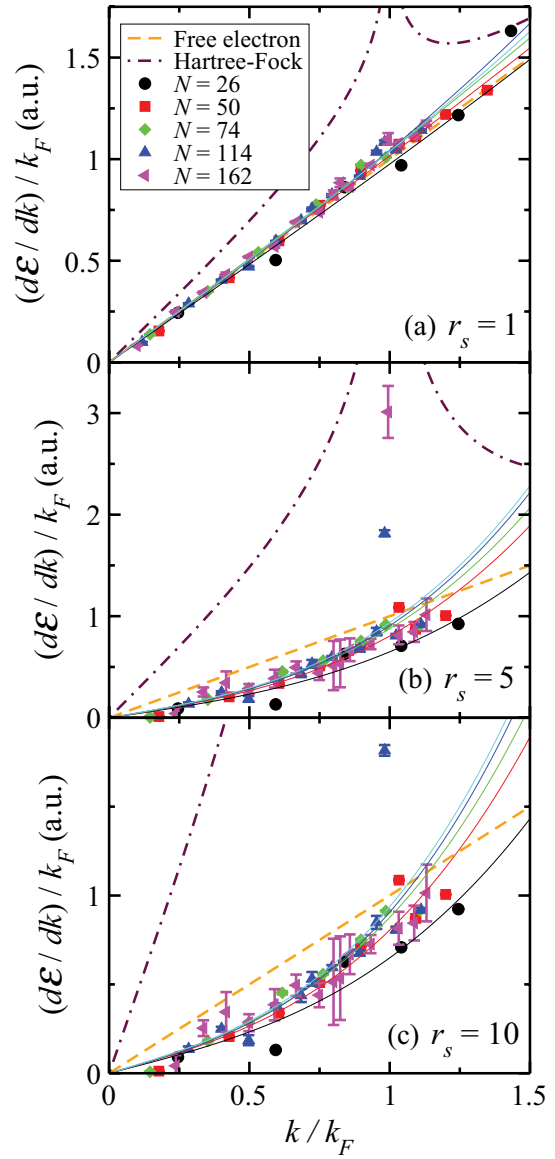

FIG. 5. (Color online) Derivatives of the paramagnetic HEG energy bands shown in Fig. 1 at (a) $r_{s}=1$, (b) $r_{s}=5$, and (c) $r_{s}=10$.

we have fitted the function

$$
m^{*}(N)=m^{*}(\infty)+b N^{-\gamma},
$$

where $m^{*}(\infty), b$, and $\gamma$ are fitting parameters, to the raw data shown in Fig. 8 and we have performed repeated fits to the data with Monte Carlo sampling of the error bars. We find that $\gamma=1.8(4), 1.4(4)$, and 1.4(3) at $r_{s}=1,5$, and 10, respectively. This indicates that the optimal exponent $\gamma$ is between 1 and 2 . This conclusion is reinforced by the results shown in Table I, where we examine the $\chi^{2}$ values of the fits and the extrapolated effective masses when different exponents are used in Eq. (4). The extrapolation shown in Fig. 7 assumes an exponent of $\gamma=3 / 2$.

\section{Quasiparticle effective mass as a function of density}

Results for the 2D HEG quasiparticle effective mass obtained by different authors, including the present work, are shown in Figs. 8 and 9 for paramagnetic and fully ferromagnetic HEGs, respectively. The present results are also given in Table II. For ferromagnetic HEGs our revised effective masses are in reasonable agreement with our previously published results. ${ }^{20}$ For paramagnetic HEGs, however, the finite-size errors are relatively large at low density, and hence finite-size extrapolation reduces the effective masses at $r_{s}=5$ and 10 by a significant amount. For paramagnetic HEGs we now find that the effective mass remains close to 1 (i.e., electron-electron

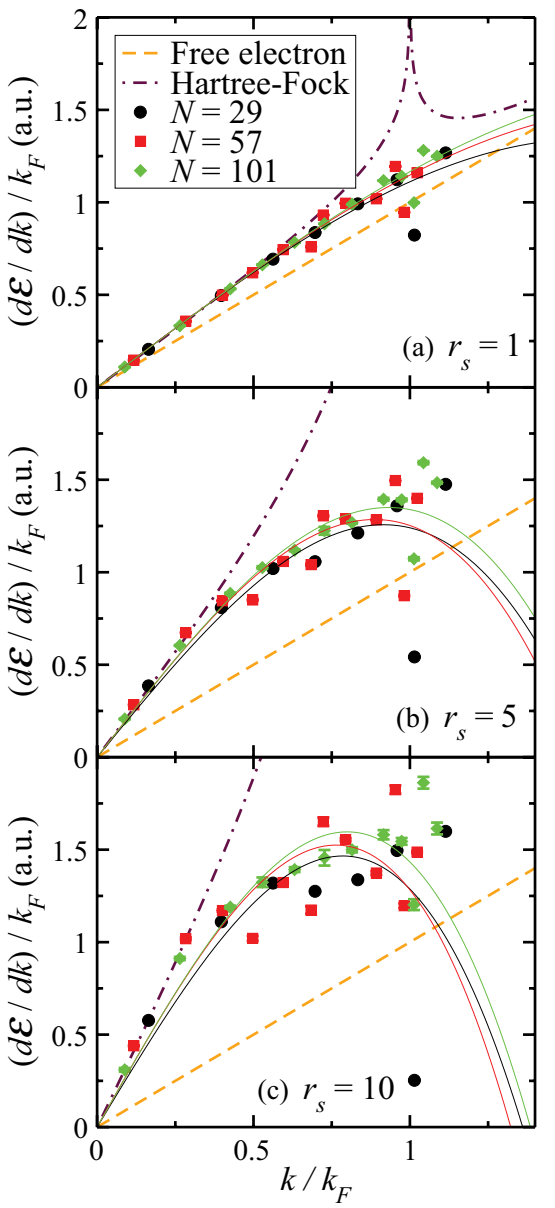

FIG. 6. (Color online) As Fig. 5, but for ferromagnetic HEGs.

interactions result in almost no renormalization of the electron mass) at all the $r_{s}$ values we have considered.

Once again we emphasize that there is no significant disagreement between the data reported in the present article and Ref. 20. The revision of the effective mass simply results from the fact that the random noise in our current data is much smaller, allowing a systematic trend with system size to be discerned and hence removed by extrapolation.

For the paramagnetic HEG, $G W$ calculations ${ }^{9,28}$ indicate a steep increase in the effective mass as the density is lowered, similar to that seen in early experiments. ${ }^{27}$ However,

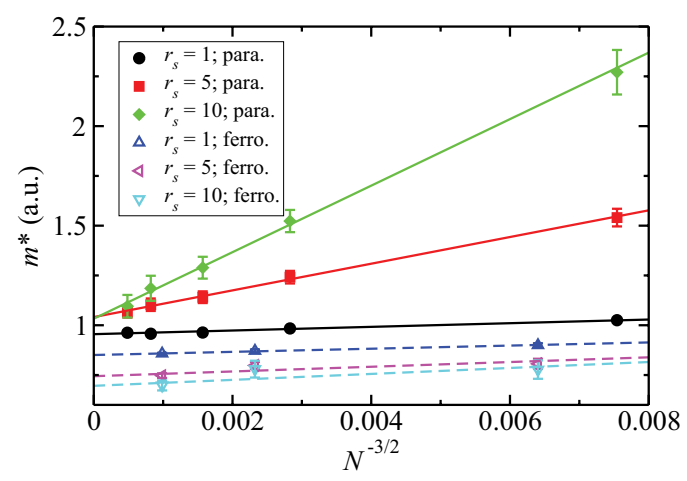

FIG. 7. (Color online) Quasiparticle effective mass $m^{*}$ against $N^{-3 / 2}$, where $N$ is the system size, for 2D HEGs. 


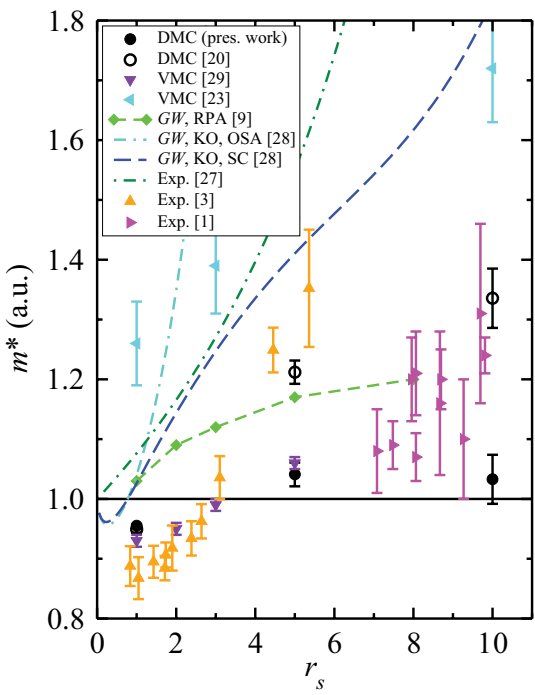

FIG. 8. (Color online) Quasiparticle effective mass $m^{*}$ against density parameter $r_{s}$ for paramagnetic or partially spin-polarized 2D HEGs, as calculated or measured by different authors. The experimental results are due to Smith and Stiles (Ref. 27), Tan et al. (Ref. 3), and Padmanabhan et al. (Ref. 1) The $G W$ results were obtained using the random-phase-approximation (RPA) effective interaction (Ref. 9) and the Kukkonen-Overhauser (KO) effective interaction (Ref. 28) by solving the Dyson equation self-consistently (SC) or within the on-shell approximation (OSA). We show the VMC results of Kwon et al. (Ref. 29) [which were later confirmed at the same system size at $r_{s}=1$ a.u. using transient-estimate DMC calculations (Ref. 30)], the VMC results of Holzmann et al. (Ref. 23) and the DMC results reported in our previous work (Ref. 20) as well as the results of the present work. All the results shown are for paramagnetic HEGs with the exception of the experimental results of Ref. 1, which are for a partially spin-polarized HEG.

the $G W$ results depend strongly on the choice of effective interaction and whether or not the calculations are performed self-consistently. At high density, e.g., $r_{s} \leqslant 1$, the $G W$ approximation might be expected to be accurate; nevertheless the disagreement between the $G W$ and QMC effective masses at $r_{s}=1$ is significant. The QMC calculations of Holzmann et $a{ }^{23}$ give quite different results from those of either Kwon et al. ${ }^{29}$ our previous work, ${ }^{20}$ or the present work. The experimental data ${ }^{1,3}$ show some evidence for enhancement of the effective mass at low density, although we do not see this in our present results.

TABLE I. Extrapolated quasiparticle effective mass $m^{*}(\infty)$ and $\chi^{2}$ value for fits to the effective-mass data as a function of system size for paramagnetic HEGs with different exponents $\gamma$ in the finite-size fitting formula [Eq. (4)].

\begin{tabular}{llllllll}
\hline \hline & \multicolumn{3}{c}{$m^{*}(\infty)$ (a.u.) } & & \multicolumn{3}{c}{$\chi^{2}$ (a.u.) } \\
\cline { 2 - 4 } \cline { 6 - 8 }$\gamma$ & $r_{s}=1$ & $r_{s}=5$ & $r_{s}=10$ & & $r_{s}=1$ & $r_{s}=5$ & $r_{s}=10$ \\
\hline $1 / 4$ & 0.88 & 0.3 & -0.7 & & 21.5 & 9.6 & 12.4 \\
$1 / 2$ & 0.924 & 0.74 & 0.3 & & 16.8 & 5.8 & 7.7 \\
1 & 0.947 & 0.97 & 0.85 & & 9.1 & 1.11 & 1.52 \\
$3 / 2$ & 0.955 & 1.04 & 1.03 & & 5.2 & 0.28 & 0.32 \\
2 & 0.959 & 1.08 & 1.13 & & 5.3 & 2.03 & 3.1 \\
\hline \hline
\end{tabular}

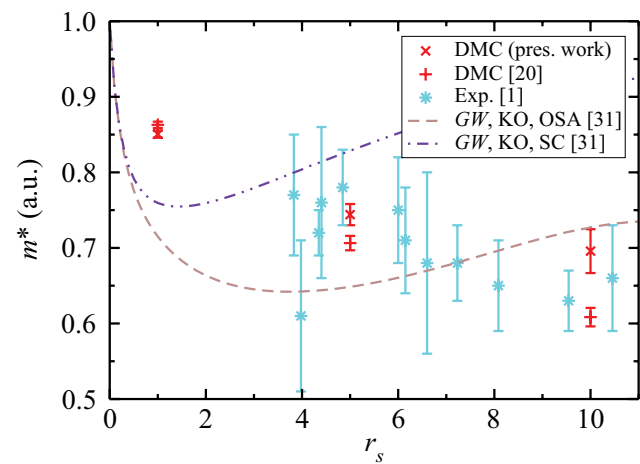

FIG. 9. (Color online) Quasiparticle effective mass $m^{*}$ against density parameter $r_{s}$ for ferromagnetic 2D HEGs. The $G W$ results were obtained using the Kukkonen-Overhauser (KO) effective interaction by solving the Dyson equation self-consistently (SC) or within the on-shell approximation (OSA) (Ref. 31). The experimental results are due to Padmanabhan et al. (Ref. 1). We show the DMC results reported in our earlier work (Ref. 20) in addition to our current results.

It should be noted that the $2 \mathrm{D}$ electron systems studied in the experiments differ from the ideal 2D HEG assumed in our calculations in that the experimental systems have finite widths and are subject to disorder. It would therefore be inappropriate to make a precise quantitative comparison of our QMC data with the existing experimental results; nevertheless we may compare the qualitative behavior.

For the ferromagnetic case, our effective-mass data are in broad agreement with the experimental results of Padmanabhan et al., ${ }^{1}$ showing a decrease in the effective mass as the density is lowered. $G W$ theory $^{31}$ also predicts a suppression of the effective mass in the range of densities considered. However, the difference between the $G W$ results obtained selfconsistently and in the on-shell approximation is significant, as is the difference with the present results, even at the highest density $\left(r_{s}=1\right)$ for which QMC data are available.

\section{CONCLUSIONS}

We have used the DMC method to calculate the singleparticle energy band and hence quasiparticle effective mass of the 2D HEG. We have achieved sufficiently high precision in our calculations that systematic finite-size errors in the quasiparticle effective mass can be observed and removed by extrapolation. This leads to a revision of the effective masses for paramagnetic HEGs at low density compared to our earlier

TABLE II. Quasiparticle effective masses for paramagnetic and fully ferromagnetic 2D HEGs, extrapolated to the thermodynamic limit.

\begin{tabular}{lrc}
\hline \hline Magnetic state & $r_{s}$ & $m^{*}($ a.u. $)$ \\
\hline Paramagnetic & 1 & $0.955(2)$ \\
Paramagnetic & 5 & $1.04(2)$ \\
Paramagnetic & 10 & $1.03(4)$ \\
Ferromagnetic & 1 & $0.851(5)$ \\
Ferromagnetic & 5 & $0.74(1)$ \\
Ferromagnetic & 10 & $0.70(3)$ \\
\hline \hline
\end{tabular}


work: ${ }^{20}$ in particular we find that there is no enhancement of the effective mass at low density.

\section{ACKNOWLEDGMENTS}

Financial support was received from Lancaster University under the Early Career Small Grant Scheme, and the
Engineering and Physical Sciences Research Council. This research used resources of the Oak Ridge Leadership Computing Facility at the Oak Ridge National Laboratory, which is supported by the Office of Science of the US Department of Energy under Contract No. DE-AC05-00OR22725. Additional computing resources were provided by the Cambridge High Performance Computing Service.
${ }^{1}$ M. Padmanabhan, T. Gokmen, N. C. Bishop, and M. Shayegan, Phys. Rev. Lett. 101, 026402 (2008).

${ }^{2}$ T. Gokmen, M. Padmanabhan, K. Vakili, E. Tutuc, and M. Shayegan, Phys. Rev. B 79, 195311 (2009).

${ }^{3}$ Y.-W. Tan, J. Zhu, H. L. Stormer, L. N. Pfeiffer, K. W. Baldwin, and K. W. West, Phys. Rev. Lett. 94, 016405 (2005).

${ }^{4}$ E. P. Wigner, Phys. Rev. 46, 1002 (1934); Trans. Faraday. Soc. 34, 678 (1938).

${ }^{5}$ B. Tanatar and D. M. Ceperley, Phys. Rev. B 39, 5005 (1989).

${ }^{6}$ F. Rapisarda and G. Senatore, Aust. J. Phys. 49, 161 (1996).

${ }^{7}$ N. D. Drummond and R. J. Needs, Phys. Rev. Lett. 102, 126402 (2009).

${ }^{8}$ L. D. Landau, Sov. Phys. JETP 3, 920 (1957); 5, 101 (1957); 8, 70 (1959).

${ }^{9}$ G. F. Giuliani and G. Vignale, Quantum Theory of the Electron Liquid (Cambridge University Press, Cambridge, 2005).

${ }^{10}$ W. M. C. Foulkes, L. Mitas, R. J. Needs, and G. Rajagopal, Rev. Mod. Phys. 73, 33 (2001).

${ }^{11}$ N. D. Drummond, M. D. Towler, and R. J. Needs, Phys. Rev. B 70, 235119 (2004).

${ }^{12}$ Y. Kwon, D. M. Ceperley, and R. M. Martin, Phys. Rev. B 48, 12037 (1993).

${ }^{13}$ P. López Ríos, A. Ma, N. D. Drummond, M. D. Towler, and R. J. Needs, Phys. Rev. E 74, 066701 (2006).

${ }^{14}$ C. J. Umrigar, K. G. Wilson, and J. W. Wilkins, Phys. Rev. Lett. 60, 1719 (1988).

${ }^{15}$ N. D. Drummond and R. J. Needs, Phys. Rev. B 72, 085124 (2005).
${ }^{16}$ C. J. Umrigar, J. Toulouse, C. Filippi, S. Sorella, and R. G. Hennig, Phys. Rev. Lett. 98, 110201 (2007).

${ }^{17}$ D. M. Ceperley and B. J. Alder, Phys. Rev. Lett. 45, 566 (1980).

${ }^{18}$ J. B. Anderson, J. Chem. Phys. 65, 4121 (1976).

${ }^{19}$ R. J. Needs, M. D. Towler, N. D. Drummond, and P. López Ríos, J. Phys.: Condens. Matter 22, 023201 (2010).

${ }^{20}$ N. D. Drummond and R. J. Needs, Phys. Rev. B 80, 245104 (2009).

${ }^{21}$ Y. Zhang and S. Das Sarma, Phys. Rev. Lett. 95, 256603 (2005).

${ }^{22}$ C. P. Weber, N. Gedik, J. E. Moore, J. Orenstein, J. Stephens, and D. D. Awschalom, Nature (London) 437, 1330 (2005).

${ }^{23}$ M. Holzmann, B. Bernu, V. Olevano, R. M. Martin, and D. M. Ceperley, Phys. Rev. B 79, 041308(R) (2009).

${ }^{24}$ M. Holzmann, B. Bernu, and D. M. Ceperley, J. Phys.: Conf. Ser. 321, 012020 (2011).

${ }^{25}$ G. Rajagopal, R. J. Needs, S. Kenny, W. M. C. Foulkes, and A. James, Phys. Rev. Lett. 73, 1959 (1994).

${ }^{26}$ G. Rajagopal, R. J. Needs, A. James, S. D. Kenny, and W. M. C. Foulkes, Phys. Rev. B 51, 10591 (1995).

${ }^{27}$ J. L. Smith and P. J. Stiles, Phys. Rev. Lett. 29, 102 (1972).

${ }^{28}$ R. Asgari and B. Tanatar, Phys. Rev. B 74, 075301 (2006).

${ }^{29}$ Y. Kwon, D. M. Ceperley, and R. M. Martin, Phys. Rev. B 50, 1684 (1994).

${ }^{30}$ Y. Kwon, D. M. Ceperley, and R. M. Martin, Phys. Rev. B 53, 7376 (1996).

${ }^{31}$ R. Asgari, T. Gokmen, B. Tanatar, M. Padmanabhan, and M. Shayegan, Phys. Rev. B 79, 235324 (2009). 